\title{
Well-Posedness for Tightly Proper Efficiency in Set-Valued Optimization Problem*
}

\author{
Yangdong $\mathrm{Xu}^{\#}$, Pingping Zhang \\ College of Mathematics and Statistics, Chongqing University, Chongqing, China \\ E-mail: \#xyd04010241@126.com,zhpp04010248@163.com \\ Received April 3, 2011; revised April 30, 2011; accepted May 10, 2011
}

\begin{abstract}
In this paper, a characterization of tightly properly efficient solutions of set-valued optimization problem is obtained. The concept of the well-posedness for a special scalar problem is linked with the tightly properly efficient solutions of set-valued optimization problem.
\end{abstract}

Keywords: Set-Valued Optimization Problem, Tightly Proper Efficiency, Well-Posedness

\section{Introduction}

One important problem in vector optimization is to find the efficient points of a set. As observed by Kuhn, Tucker and later by Geoffrion, some efficient points exhibit certain abnormal properties. To eliminate such abnormal efficient points, various concept of proper efficiency have been introduced. The original concept was introduced by Kuhn and Tucker [1] and Geoffrion [2], and later modified and formulated in a more generalized framework by Borwein [3], Hartley [4], Benson [5], Henig [6], Borwein and Zhuang [7]; also see the references there in. Particularly, the concept of tightly proper efficiency was introduced by Zaffaroni [8], and he used a special scalar function to characterize the tightly proper efficiency, and obtained some properties of tightly proper efficiency.

In this paper, we study the characterization and wellposedness for tightly proper efficiency in set-valued vector optimization problem. The paper is organized as follows. In Section 2, some concepts of tightly proper efficiency and some preliminary results are given. In Section 3, the characterization and well-posedness for tightly proper efficiency in set-valued vector optimization problem is discussed.

\section{Preliminaries}

Throughout this paper, let $X$ be a linear space, $Y$ and $Z$ be two finite dimensional, with topological dual

\footnotetext{
${ }^{*}$ This research was partially supported by the National Natural Science Foundation of China (Grant number: 10871216).
}

spaces $Y^{*}$ and $Z^{*}$. For a set $A \subset Y, c l A$, int $A, \partial A$, and $A^{c}$ denote the closure, the interior, the boundary and the complement of $A$, respectively. Moreover, we will denote with $B$ the closed unit ball of $Y$. A set $C \subset Y$ is said to be a cone if $\lambda c \in C$ for any $c \in C$ and $\lambda \geq 0$. A cone $C$ is said to be convex if $C+C \subset C$, and it is said to be pointed if $C \cap(-C)=\{0\}$. In the sequel we suppose that $C \subset Y$ is a convex, closed, pointed cone with nonempty interior. We say that the set $\Theta \subset Y$ is a base for $C$ if $\Theta$ is convex with $0 \notin c l C$ and $C \backslash\{0\}=$ cone $\Theta=\{y \in Y: y=\lambda \theta, \lambda>0, \theta \in \Theta\}$.

Definition 2.1: A point $\bar{y} \in S \subset Y$ is said to be efficient with respect to $C$ (denoted $\bar{y} \in E(S, C)$ ) if

$$
(S-\bar{y}) \cap-C=\left\{0_{Y}\right\}
$$

Definition 2.2: [8] The point $\bar{y} \in S \subset Y$ is called tightly proper efficient with respect to $C$ (denoted $\bar{y} \in \operatorname{TPE}(S, C))$ if there exists an open convex set $K \subset Y$ with $0_{Y} \in \partial K$ satisfying $(S-\bar{y}) \cap K=\varnothing$ and there exists $\delta>0$ such that

$$
K^{c} \cap(\delta B-C) \subseteq B
$$

It is easy to verify that

$$
T P E(S, C) \subset E(S, C)
$$

Definition 2.3: [9] Let $S$ be a nonempty subset of $Y$. The contingent cone (or the Bouligand tangent cone) to $S$ at $y_{0} \in c l S$ is the set

$$
\begin{aligned}
T\left(S, y_{0}\right):= & \left\{v \in Y: \exists\left\{\lambda_{n}\right\} \subset R^{+}, \exists\left\{y_{n}\right\} \subseteq Y,\right. \\
& \left.y_{n} \rightarrow y_{0} \text { with } v=\lim _{n \rightarrow \infty} \lambda_{n}\left(y_{n}-y_{0}\right)\right\}
\end{aligned}
$$


Jahn [9] have gotten the following proposition on the contingent cone to $S$ at $y_{0} \in c l S$.

Proposition 2.1: [9] Let $S$ be a nonempty convex subset of a real normed space. Then

$$
T(S, C)=\operatorname{clcone}(S-\bar{y})
$$

\section{Tightly Proper Efficiency and Well-Posedness}

Consider the following vector optimization problem with set-valued maps:

(VP) $\min F(x)$,

s.t. $G(x) \cap(-D) \neq \varnothing, x \in X$,

where $F: X \rightarrow 2^{Y}$ and $G: X \rightarrow 2^{Z}$ are set-valued maps, respectively. $D$ is a closed,convex, pointed cone of $Z$.

Denote the feasible solution set of (VP) by

$$
A:=\{x \in X: G(x) \cap 9-D \neq \varnothing\}
$$

and the image of $A$ under $F$ by

$$
F(A)=\bigcup_{x \in A} F(x)
$$

Definition 3.1: A point $\bar{x}$ is said to be a tightly proper efficient solution of $(V P)$, if there exists $\bar{y} \in F(\bar{x})$ such that $\bar{y} \in \operatorname{TPE}[F(A), C]$, and the point $(\bar{x}, \bar{y})$ is said to be a tightly properly efficient minimizer of $(V P)$.

Definition 3.2: For a set $S \subseteq Y$. Let the function $\Delta_{S}: Y \rightarrow R \cup\{ \pm \infty\}$ be defined as

$$
\Delta_{S}(y)=d_{S}(y)-d_{Y \backslash S}(y)
$$

where $d_{S}(y)=\inf \{\|s-y\|: s \in S\}$ with $d_{\varnothing}(y)=+\infty$.

The function $\Delta_{S}$ was introduced in [8], its main properties are gathered together in the following proposition.

Proposition 3.1: [8] Let $C$ be a convex cone with nonempty interior, then the function $\Delta_{C}$ is convex, positively homogenous and lipschitzian. Moreover, this function is negative on the interior of $C$, null on $\partial C$ and positive on int $C^{c}$.

We consider the parameterized scalar problem:

$$
\begin{gathered}
\left(P_{\bar{y}}\right) \min \Delta_{-C}(y-\bar{y}) \\
\text { s.t. } \quad y \in F(A)
\end{gathered}
$$

where $\bar{y} \in Y$.

Definition 3.3: Let $\bar{y} \in Y$, the parameterized scalar optimization problem $\left(P_{\bar{y}}\right)$ is Tikhonov well-posed if

1) $\Delta_{-C}(y-\bar{y})=d_{-C}(y-\bar{y})>0$ for all $y \in F(A)$ with $y \neq \bar{y}$;

2) for all $\left\{y_{n}\right\} \subseteq F(A)$ with $\quad d_{-C}\left(y_{n}-\bar{y}\right) \rightarrow 0$ implies that $y_{n} \rightarrow \bar{y}$.
We denote by

$W P V P=\left\{y \in F(A) \mid P_{y}\right.$ is Tikhonov well posed $\}$.

Lemma 3.1: [8] Consider the following statements:

(a) the point $\bar{y}$ is a tightly properly efficient point in $S$;

(b) there exists an open convex set $K \subset Y$ such that $-C \backslash\left\{0_{Y}\right\} \subseteq K$ and $(S-\bar{y}) \cap K=\varnothing$;

(c) $T(S+C, \bar{y}) \cap-C=\left\{0_{Y}\right\}$.

If $Y$ is a any normed space, then it holds that $(a) \Rightarrow(b) \Rightarrow(c)$. If $Y$ is finite dimensional, we have also that $(c) \Rightarrow(a)$ and all statements are equivalent.

The following theorem characterize the relation between tightly properly efficient points of(VP) and the parameterized scalar problem $\left(P_{\bar{y}}\right)$.

Theorem 3.1: Let $\bar{x} \in A, \bar{y} \in F(\bar{x})$. The $(\bar{x}, \bar{y})$ is a tightly properly efficient minimizer of $(V P)$, then $\bar{y}$ is a solution of $\left(P_{\bar{y}}\right)$.

Proof. We show that $\bar{y}$ is a solution of the scalar problem $\left(P_{\bar{y}}\right)$. Indeed, by Proposition 3.1 and Lemma 3.1(b), we have

$$
\Delta_{-C}(y-\bar{y})=d_{-C}(y-\bar{y}) \geq 0, \forall y \in F(A)
$$

Noting that $d_{-C}(y-\bar{y})=0$, thus we have that $\bar{y}$ is just the solution of the problem $\left(P_{\bar{y}}\right)$.

Remark 3.1: The converse of Theorem 3.1 may be not valid, the following example can illustrate the case.

Example 3.1: Let $X=R, Y=R^{2}$ and $Z=R$. Given $C=R_{+}^{2}, D=R_{+}$.

$F(x)= \begin{cases}\left\{(x, y) \subset R \times R_{+} \mid y \geq 1-\sqrt{1-(x-1)^{2}}\right\}, & \text { if } x \in[0,1] \\ \left\{(x, y) \subset R \times R_{+}\right\}, & \text {otherwise }\end{cases}$

$g(x)=[-x,-x+1]$, for any $x \in X$

Thus, the feasible set of (VP)

$$
A=\left\{x \in X \mid G(x) \cap\left(-R_{+}\right) \neq \varnothing\right\}=[0,+\infty)
$$

The set of $F(A)$ can see Figure 1.

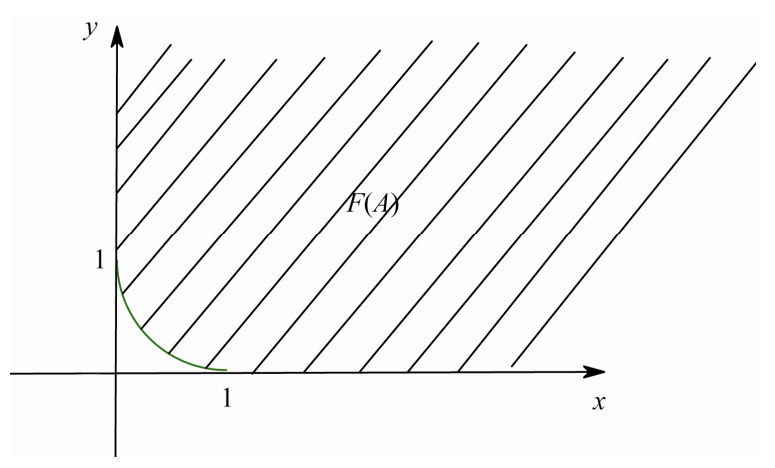

Figure 1. The set of $F(A)$. 
$\bar{y}=(1,0) \in Y$ is a solution of $P_{\bar{y}}$, but $(1,(1,0))$ is not a tightly properly efficient minimizer of (VP). Thus, the converse of Theorem 3.1 is not valid.

Theorem 3.2: Let $\bar{x} \in A, \bar{y} \in F(\bar{x})$. Then, the $(\bar{x}, \bar{y})$ is a tightly properly efficient minimizer of $\left(P_{\bar{y}}\right)$ if and only if the scalar problem $\left(P_{\bar{y}}\right)$ is Tikhonnov well-posed.

Proof. If $(\bar{x}, \bar{y})$ is a tightly properly efficient minimizer of $\left(P_{\bar{y}}\right)$, let us show that the scalar problem $\left(P_{\bar{y}}\right)$ is Tikhonnov well-posed. We argue by contradiction: if the conclusion is false, then there exists a sequence $\left\{y_{n}\right\} \subseteq F(A)$ such that $y_{n}-\bar{y} \notin \varepsilon B$ for some $\varepsilon>0$ and $d_{-C}\left(y_{n}-\bar{y}\right) \rightarrow 0$, which means that there exists a sequence $\left\{c_{n}\right\} \subseteq C$ with $y_{n}+c_{n}-\bar{y} \rightarrow 0$ and $c_{n} \notin\left(\frac{\varepsilon}{2}\right) B$. Since we can always write $c_{n}=\lambda_{n} \theta_{n}$ with $\theta_{n} \in \Theta$, it follows that $\lambda_{n}$ does not converge to zero, i.e., there exists a subsequence (we again call it $\lambda_{n}$ ) with $\lambda_{n}>\beta$ for some $\beta>0$. Now take $\alpha_{n}=\left\|y_{n}+c_{n}-\bar{y}\right\|^{\frac{1}{2}}$, since $\lambda_{n}$ is bounded away from zero and $\alpha_{n} \rightarrow 0$, thus we have $\alpha_{n}<\lambda_{n}$. Set $c_{n}^{\prime}=\left(\lambda_{n}-\alpha_{n}\right) \theta_{n}$ to obtain $y_{n}+c_{n}^{\prime}-\bar{y}=y_{n}+c_{n}-\bar{y}-\alpha_{n} \theta_{n} \rightarrow 0$ and $\alpha_{n}^{-1}\left(y_{n}+c_{n}^{\prime}-\bar{y}\right) \rightarrow-\theta \neq 0$. And by the Lemma 3.1, we get contradiction, therefore it shows that $\left(P_{\bar{y}}\right)$ is Tikhonov well-posed.

Conversely, we argue by contradiction: if the conclusion is false, then by $Y$ is a finite dimensional real normal space and Lemma 3.1(c), there exists $v \in-C \backslash\left\{0_{Y}\right\}$ such that $v \in T(S+C, \bar{y})$. Thus there exist $\left\{y_{n}\right\} \subseteq F(A),\left\{c_{n}\right\} \subseteq C$ with $y_{n}+c_{n} \rightarrow \bar{y}$ and $\left\{\beta_{n}\right\} \subseteq R_{+}$such that

$$
v=\lim _{n \rightarrow \infty} \beta_{n}\left(y_{n}+c_{n}-\bar{y}\right)
$$

It follows that

$\Delta_{-C}\left(\beta_{n}\left(y_{n}-\bar{y}\right)\right)=\Delta_{-C}\left(\beta_{n}\left(y_{n}+c_{n}-\bar{y}\right)\right) \rightarrow 0$. Since

$\left(P_{\bar{y}}\right)$ is Tikhonov well-posed, we have

$$
\beta_{n}\left(y_{n}-\bar{y}\right) \rightarrow 0
$$

By (1), we have $\beta_{n} c_{n} \rightarrow v$, but since $C$ is pointed and $v \in-C \backslash\left\{0_{Y}\right\}$. Therefore it is a contradiction.

We give the following example to illustrate Theorem

\section{2 .}

Example 3.2: In this example, we shall continue to consider Example 3.1. By Definition 3.1 and Definition 3.3, we can get

$$
\begin{aligned}
& \operatorname{TPE}(F((A), C)) \\
& =\left\{(x, x, y) \mid y=1-\sqrt{1-(x-1)^{2}}, x \in(0,1)\right\}
\end{aligned}
$$

and

$$
W P V P=\left\{(x, y) \mid y=1-\sqrt{1-(x-1)^{2}}, x \in(0,1)\right\}
$$

Therefore, Theorem 3.2 is valid.

\section{References}

[1] H. W. Kuhn and A. W. Tucker, "Nonlinear Programming," Proceedings of the 2nd Berkeley Symposium on Mathematical Statistics and Probability, Berkele, 31 July-12 August 1950, pp. 481-492.

[2] A. M. Geoffrion, "Proper Efficiency and the Theory of Vector Maximization," Journal of Mathematical Analysis and Applications, Vol. 22, No. 3, 1968, pp. 618-630.

[3] J. M. Borwein, "Proper Efficient Points for Maximizations with Respect to Cones," SIAM Journal on Control and Optimization, Vol. 15, No. 1, 1977, pp. 57-63. doi: $10.1137 / 0315004$

[4] R. Hartley, "On Cone Efficiency, Cone convexity, and Cone Compactness," SIAM Journal on Applied Mathematics, Vol. 34, No. 1, 1978, pp. 211-222. doi: $10.1137 / 0134018$

[5] H. P. Benson, "An Improved Definition of Proper Efficiency for Vector Maximization with Respect to Cones," Journal of Mathematical Analysis and Applications, Vol. 71 , No. 1, 1979, pp. 232-241. doi:10.1016/0022-247X(79)90226-9

[6] M. I. Henig, "Proper Efficiency with Respect to Cones," Journal of Optimization Theory and Applications, Vol. 36, No. 3, 1982, pp. 387-407. doi:10.1007/BF00934353

[7] J. M. Browein and D. Zhuang, "Superefficiency in Vector Optimization," Transactions of the American Mathematical Society, Vol. 338, No. 1, 1993, pp. 105-122. doi: $10.2307 / 2154446$

[8] A. Zaffaroni, "Degrees of Efficiency and Degrees of Minimality," SIAM Journal on Control and Optimization, Vol. 42, No. 3, 2003, pp. 1071-1086. doi:10.1137/S0363012902411532

[9] J. Jahn, "Vector Optimization," Springer-Verlag, Berlin, 2003. 